\title{
Mixed-State Entanglement from Local Randomized Measurements
}

\author{
Andreas Elben $\odot,{ }^{1,2, *}$ Richard Kueng, ${ }^{3, *}$ Hsin-Yuan (Robert) Huang $\odot,{ }^{4,5}$ Rick van Bijnen $\odot,{ }^{1,2}$ \\ Christian Kokail, ${ }^{1,2}$ Marcello Dalmonte, ${ }^{6,7}$ Pasquale Calabrese, ${ }^{6,7,8}$ Barbara Kraus $\odot,{ }^{9}$ \\ John Preskill@, ${ }^{4,5,10,11}$ Peter Zoller, ${ }^{1,2}$ and Benoît Vermersch $\oplus^{1,2,12}$ \\ ${ }^{1}$ Center for Quantum Physics, University of Innsbruck, Innsbruck A-6020, Austria \\ ${ }^{2}$ Institute for Quantum Optics and Quantum Information of the Austrian Academy of Sciences, Innsbruck A-6020, Austria \\ ${ }^{3}$ Institute for Integrated Circuits, Johannes Kepler University Linz, Altenbergerstrasse 69, 4040 Linz, Austria \\ ${ }_{4}^{4}$ Institute for Quantum Information and Matter, Caltech, Pasadena, California 91125, USA \\ ${ }^{5}$ Department of Computing and Mathematical Sciences, Caltech, Pasadena, California 91125, USA \\ ${ }^{6}$ The Abdus Salam International Center for Theoretical Physics, Strada Costiera 11, 34151 Trieste, Italy \\ ${ }^{7}$ SISSA, via Bonomea 265, 34136 Trieste, Italy \\ ${ }^{8}$ INFN, via Bonomea 265, 34136 Trieste, Italy \\ ${ }^{9}$ Institute for Theoretical Physics, University of Innsbruck, A6020 Innsbruck, Austria \\ ${ }^{10}$ Walter Burke Institute for Theoretical Physics, Caltech, Pasadena, California 91125, USA \\ ${ }^{11}$ AWS Center for Quantum Computing, Pasadena, California 91125, USA \\ ${ }^{12}$ Université Grenoble Alpes, CNRS, LPMMC, 38000 Grenoble, France
}

(Received 22 July 2020; accepted 20 October 2020; published 11 November 2020)

\begin{abstract}
We propose a method for detecting bipartite entanglement in a many-body mixed state based on estimating moments of the partially transposed density matrix. The estimates are obtained by performing local random measurements on the state, followed by postprocessing using the classical shadows framework. Our method can be applied to any quantum system with single-qubit control. We provide a detailed analysis of the required number of experimental runs, and demonstrate the protocol using existing experimental data [Brydges et al., Science 364, 260 (2019)].
\end{abstract}

DOI: 10.1103/PhysRevLett.125.200501

Engineered quantum many-body systems exist in today's laboratories as noisy intermediate scale quantum devices (NISQ) [1]. This provides us with novel opportunities to study and quantify entanglement—a fundamental concept in both quantum information theory [2] and many-body quantum physics $[3,4]$. For pure (or nearly pure) states, entanglement has been detected by measuring the second Rényi entropy [5-10]. This has been achieved via, for instance, many-body quantum interference [7-9,11,12] (see also Refs. $[13,14]$ ) and randomized measurements [10,15-18]. However, many states of interest are actually highly mixed-either because of decoherence, or because they describe interesting subregions of a larger, globally entangled, system. Developing protocols which detect and quantify mixed-state entanglement on intermediate scale quantum devices is thus an outstanding challenge.

Below we propose and experimentally demonstrate conditions for mixed-state entanglement and measurement protocols based on the positive partial transpose (PPT) condition [2,5,19]. Consider two partitions $A$ and $B$ described by a (reduced) density matrix $\rho_{A B}$. The wellknown PPT condition checks if the partially transposed (PT) density matrix $\rho_{A B}^{T_{A}}$ [20] is positive semidefinite, i.e., all eigenvalues are non-negative. If the PPT condition is violated-i.e., $\rho_{A B}^{T_{A}}$ does have negative eigenvalues- $A$ and $B$ must be entangled. It is possible to turn the PPT condition into a quantitative entanglement measure. The negativity $\mathcal{N}\left(\rho_{A B}\right)=\sum_{\lambda<0}|\lambda|$, with $\lambda$ the spectrum of $\rho_{A B}^{T_{A}}$, is positive if and only if the underlying state $\rho_{A B}$ violates the PPT condition [21]. While applicable to mixed states, computing the negativity requires accurately estimating the full spectrum of $\rho_{A B}^{T_{A}}$. We bypass this challenge by considering moments of the partially transposed density matrix (PT moments) instead:

$$
p_{n}=\operatorname{Tr}\left[\left(\rho_{A B}^{T_{A}}\right)^{n}\right] \quad \text { for } n=1,2,3, \ldots
$$

These have been first studied in quantum field theory to quantify correlations in many-body systems [22]. Clearly, $p_{1}=\operatorname{tr}\left(\rho_{A B}\right)=1$, while $p_{2}$ is equal to the purity $\operatorname{tr}\left[\rho_{A B}^{2}\right]$ (see Table I in the Supplemental Material [23] for a visual derivation). Hence, $p_{3}$ is the lowest PT moment that captures meaningful information about the partial transpose (see also Ref. [29]).

In this Letter, we first show that the first three PT moments can be used to define a simple yet powerful test for bipartite entanglement:

$$
\rho_{A B} \in \mathrm{PPT} \Rightarrow p_{3} \geq p_{2}^{2} \text {. }
$$


(a)

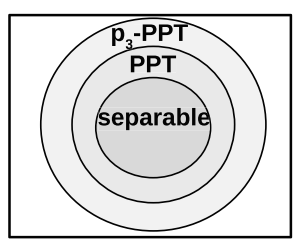

(c)

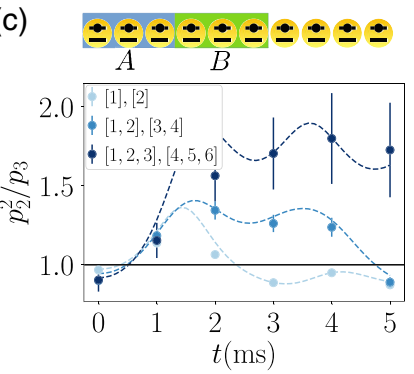

(b)

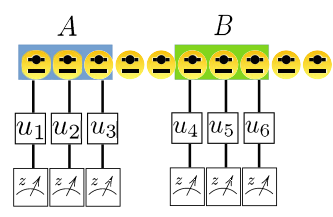

(d)

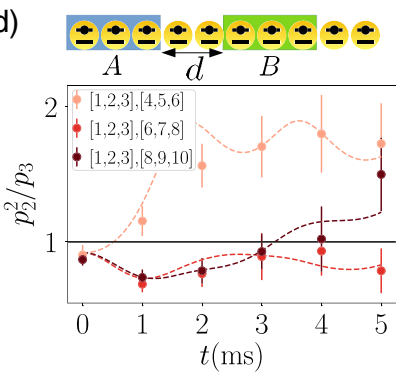

FIG. 1. Protocol and illustrations. (a) The $p_{3}$-PPT condition can be used to demonstrate mixed-state bipartite entanglement with PT moments. Separable states are PPT states and also fulfill the $p_{3}$-PPT condition. Thus, quantum states which violate the $p_{3}$-PPT condition must be bipartite entangled [see also Eq. (2)]. (b) In our protocol, PT moments are measured by applying local random unitaries followed by computational basis measurements. (c), (d) Violation of the $p_{3}$-PPT condition, i.e., $p_{2}^{2}>p_{3}$, is experimentally observed for connected (c) and disconnected (separated by $d=0,2,4$ spins) (d) partitions $A$ and $B$ at various times $t$ after a quantum quench [10]. Dots: experimental results. Error bars: Jackknife estimates of statistical errors. Lines: numerical simulations including the decoherence model presented in Ref. [10].

The $p_{3}-P P T$ condition is the contrapositive of this assertion: if $p_{3}<p_{2}^{2}$, then $\rho_{A B}$ violates the PPT condition [see Fig. 1(a)] and must therefore be entangled (see Supplemental Material [23] for the proof). Similar to the PPT condition, the $p_{3}$-PPT condition applies to mixed states and is completely independent of the state in question. This is a key distinction from entanglement witnesses [30,31], which can be more powerful, but which usually require detailed prior information about the state. While in general weaker than the full PPT condition, the $p_{3}$-PPT condition relies on comparing two comparatively simple functionals and outperforms other state-independent entanglement detection protocols, like comparing purities of various nested subsystems [5,7-10,23]. As shown in the Supplemental Material [23], the $p_{3}$-PPT condition becomes equivalent to the PPT condition for Werner states (in this case, it is a necessary and sufficient condition for bipartite entanglement [32]).

The second main contribution of this Letter is a measurement protocol to determine PT moments in NISQ devices. Crucially, we employ randomized measurements implemented with local (single-qubit) random unitaries, see Fig. 1(b), which are readily available in NISQ devices and have been already successfully applied to measure entanglement entropies, many-body state fidelities, and

out-of-time ordered correlators [10,33-35]. In contrast to previous proposals for measuring PT moments, our protocol does not rely on many-body interference between identical state copies $[6,29,36]$, or on using global entangling random unitaries [37] built from interacting Hamiltonians [16,38-40]. Instead, it only requires singlequbit control, and allows for the estimation of many distinct PT moments from the same data. In particular, arbitrary orders $n \geq 2$ and arbitrary (connected, as well as disconnected) partitions $A, B$ can be measured.

While the experimental setup for our measurement protocol is reminiscent of quantum state tomography [41-44], there are fundamental differences regarding the required number of measurements (as independent state copies), and the way the measured data is processed. Without strong assumptions on the state $[42,43]$, performing tomography to infer an $\epsilon$-approximation of an unknown density matrix $\rho_{A B}$ (e.g., in order to subsequently compute $\epsilon$ approximations of $p_{n}$ ) requires (at least) order $2^{|A B|} \operatorname{rank}\left(\rho_{A B}\right) / \epsilon^{2}$ measurements $[45,46]$. In the high accuracy regime $(\epsilon \ll 1)$, our direct estimation protocol instead only requires order $2^{|A B|} / \epsilon^{2}$ measurements. For highly mixed states - the central topic of this work-this discrepancy heralds a significant reduction in measurement resources. Furthermore, we predict PT-moments through a "direct" and (multi-) linear postprocessing of the measurement data represented as "classical shadows" [18]. Thus, data processing is cheap-both in memory and runtime - and can be massively parallelized. Similar to previous measurement $[10,15,16,18,34,35,47-49]$ and entanglement detection [50-54] protocols based on randomized measurements, this is another distinct advantage over tomography which typically requires expensive data-processing algorithms [41] or training a neural network [43].

Finally, we demonstrate our measurement protocol and the $p_{3}$-PPT condition experimentally in the context of the quantum simulation of many-body systems. Here, PT moments have been shown to reveal universal properties of quantum phases of matter [22,55-58] and their transitions $[22,55,59,60]$. Out of equilibrium, PT moments allow to understand the dynamical process of thermalization [61-64], and the fate of (many-body) localization in presence of decoherence [65]. In this work, we analyze the data of Ref. [10] corresponding to the out-of-equilibrium dynamics in a spin model with long-range interactions, which was implemented in a 10-qubit trapped ion quantum simulator. In particular, we certify the presence of mixed-state entanglement via the $p_{3}$-PPT condition [see Figs. 1(c)-1(d), and for details below]. Furthermore, we monitor the time evolution of $p_{3}$ and observe dynamical signatures of entanglement spreading and thermalization $[61,62]$.

Protocol.-The experimental ingredients to measure PT moments build on resources similar to the ones presented in Ref. [16] and realized in Ref. [10] to measure Rényi 
entropies. The key new element is the postprocessing of the experimental data [18]. As shown in Fig. 1, the quantum state of interest is realized in a system of $N$ qubits. In the partitions $A$ and $B$, consisting of $|A|$ and $|B|$ spins, respectively, a randomized measurement is performed by applying random local unitaries $u=u_{1} \otimes \ldots \otimes u_{|A B|}$, with $u_{i}$ independent single qubit rotation sampled from a unitary three design $[38,66]$, and a subsequent projective measurement in the computational basis with outcome $\mathbf{k}=\left(k_{1}, \ldots, k_{|A B|}\right)$. This is subsequently repeated with $M$ different random unitaries such that a dataset of $M$ bit strings $\mathbf{k}^{(r)}$ with $r=1, \ldots, M$ is collected.

From this dataset, the PT moments $p_{n}$ can be estimated without having to reconstruct the density matrix $\rho_{A B}$, and with a significantly smaller number of experimental runs $M$ than required for full quantum state tomography. To obtain such estimates, we rely on two observations. First, each outcome $\mathbf{k}^{(r)}$ can be used to define an unbiased estimator

$$
\hat{\rho}_{A B}^{(r)}=\bigotimes_{i \in A B}\left[3\left(u_{i}^{(r)}\right)^{\dagger}\left|k_{i}^{(r)}\right\rangle\left\langle k_{i}^{(r)}\right| u_{i}^{(r)}-\mathbb{I}_{2}\right]
$$

of the density matrix $\rho_{A B}$, i.e., $\mathbb{E}\left[\hat{\rho}_{A B}^{(r)}\right]=\rho_{A B}$ with the expectation value taken over the unitary ensemble and projective measurements $[17,18,67,68]$. Second, the PT moments $p_{n}$ can be viewed as an expectation value of an $n$-copy observable $\vec{\Pi}_{A} \overleftarrow{\Pi}_{B}$ evaluated on $n$ copies of the original density matrix $\rho_{A B}$,

$$
p_{n}=\operatorname{Tr}\left[\vec{\Pi}_{A} \overleftarrow{\Pi}_{B} \rho_{A B}^{\otimes n}\right] .
$$

Here, $\vec{\Pi}_{A}$ and $\overleftarrow{\Pi}_{B}$ are $n$-copy cyclic permutation operators $\quad \vec{\Pi}_{A}\left|\mathbf{k}_{A}^{[1]}, \mathbf{k}_{A}^{[2]}, \ldots, \mathbf{k}_{A}^{[n]}\right\rangle=\left|\mathbf{k}_{A}^{[n]}, \mathbf{k}_{A}^{[1]}, \ldots, \mathbf{k}_{A}^{[n-1]}\right\rangle$, $\overleftarrow{\Pi}_{B}\left|\mathbf{k}_{B}^{[1]}, \mathbf{k}_{B}^{[2]}, \ldots, \mathbf{k}_{B}^{[n]}\right\rangle=\left|\mathbf{k}_{B}^{[2]}, \ldots, \mathbf{k}_{B}^{[n]}, \mathbf{k}_{B}^{[1]}\right\rangle$ that act on the partitions $A$ and $B$, respectively.

Estimators of the PT moments $p_{n}$ can now be derived from Eqs. (3) and (4) using $U$ statistics [69]. Replacing $\rho^{\otimes n}$ with $\hat{\rho}^{\left(r_{1}\right)} \otimes \cdots \otimes \hat{\rho}^{\left(r_{n}\right)}$, where $r_{1} \neq r_{2} \neq \cdots \neq r_{n}$, corresponding to independently sampled random unitaries $u^{\left(r_{1}\right)}, \ldots, u^{\left(r_{n}\right)}$, we define the $U$ statistics

$\hat{p}_{n}=\frac{1}{n !}\left(\begin{array}{c}M \\ n\end{array}\right)^{-1} \sum_{r_{1} \neq r_{2} \neq \cdots \neq r_{n}} \operatorname{Tr}\left[\vec{\Pi}_{A} \overleftarrow{\Pi}_{B} \hat{\rho}_{A B}^{\left(r_{1}\right)} \otimes \cdots \otimes \hat{\rho}_{A B}^{\left(r_{n}\right)}\right]$.

It follows from the defining properties of $U$ statistics that $\hat{p}_{n}$ is an unbiased estimator of $p_{n}$, i.e., $\mathbb{E}\left[\hat{p}_{n}\right]=p_{n}$ with the expectation value taken over the unitary ensemble and projective measurements [69]. Its variance governs the statistical errors arising from finite $M$. Furthermore, a quick inspection of Eqs. (3) and (4) reveals that the summands in Eq. (5) completely factorize into contractions of single qubit matrices, $\operatorname{Tr}\left[\vec{\Pi}_{A} \overleftarrow{\Pi}_{B} \hat{\rho}_{A B}^{\left(r_{1}\right)} \otimes \cdots \otimes \hat{\rho}_{A B}^{\left(r_{n}\right)}\right]=$ $\Pi_{i \in A} \operatorname{Tr}\left[\hat{\rho}_{i}^{\left(r_{1}\right), T} \cdots \hat{\rho}_{i}^{\left(r_{n}\right), T}\right] \Pi_{i \in B} \operatorname{Tr}\left[\hat{\rho}_{i}^{\left(r_{1}\right)} \cdots \hat{\rho}_{i}^{\left(r_{n}\right)}\right]$, with $\hat{\rho}_{A B}^{(r)}=$ $\otimes_{i \in A B} \hat{\rho}_{i}^{(r)}$ as in Eq. (3). Thus, given $M$ observed bit strings $\mathbf{k}_{r}$, one can determine $\hat{p}_{n}$ with classical data processing scaling as $M^{n}|A B|$, without storing exponentially large matrices on the classical post-processing device.

Statistical errors.-As demonstrated in Figs. 1(c), 1(d), PT moments can be inferred using a finite number of experimental runs $M$. Here, we investigate in detail the statistical errors arising from the finite value of $M$.

Analytically, we bound statistical errors based on the variance of the multicopy observable in question. For $p_{2}=\operatorname{Tr}\left[\left(\rho_{A B}^{T_{A}}\right)^{2}\right]$, our analysis reveals that the error decay rate depends on number of measurements $M$. In the large $M$ regime, the error is proportional to $2^{|A B|} p_{2} / \sqrt{M}$. This error bound is multiplicative-i.e., the size of the error is proportional to the size of the target $p_{2}$-and $1 / \sqrt{M}$ captures the expected decay rate for an estimation procedure that relies on empirical averaging. For small and intermediate values of $M$, the estimation error is instead bounded by $8 \times 2^{1.5|A B|} / M$. While this is worse in terms of constants, the error decays at a much faster rate proportional to $1 / M$. Qualitatively similar results apply for estimating $p_{3}=\operatorname{Tr}\left[\left(\rho_{A B}^{T_{A}}\right)^{3}\right]$, but there can be three decay regimes. For large $M$, the estimation error is bounded by $2^{|A B|} p_{2}^{2} / \sqrt{M}$. This again captures the asymptotically optimal rate $1 / \sqrt{M}$ associated with empirical averaging, but the constant is suppressed by $p_{2}^{2}$, not $p_{3}$ itself. For intermediate $M$, the error decay rate is proportional to $1 / M$, while an even faster rate $\propto 1 / M^{3 / 2}$ governs the error decay for small $M$. We refer to the Supplemental Material [23] for detailed statements and proofs.

Now, we test these predictions numerically by simulating the experimental protocol for various values of $M$ in systems with $N=|A B|$ qubits where a pure GHZ state $\rho=\left|\phi_{\mathrm{GHZ}}\right\rangle\left\langle\phi_{\mathrm{GHZ}}\right|$ is prepared. Here, $A$ corresponds to the first $N / 2$ qubits, and $B$ is the complement. The results are shown in Fig. 2 and support our analytical error bounds. They highlight in particular that the number of measurement repetitions necessary to achieve a desired accuracy of $\sim 0.1$ scales as $2^{|A B|}$. This enables the estimation of PT moments in state of the art platforms with high repetition rates. These findings are discussed and confirmed for the ground state of the transverse Ising model in the Supplemental Material [23].
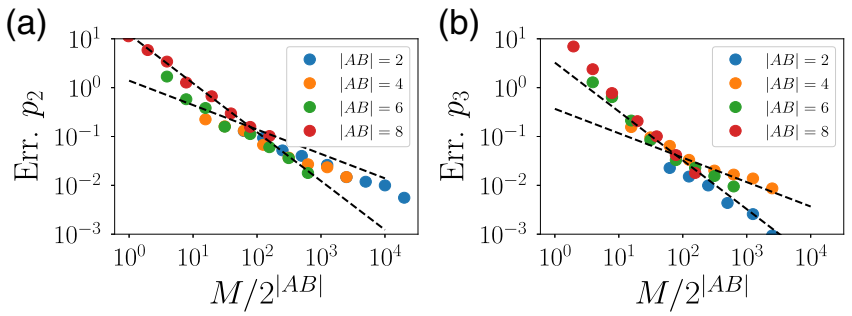

FIG. 2. Statistical errors for the GHZ state. Dashed lines represent scalings of $\propto 1 / M$, and $\propto 1 / \sqrt{M}$. In both cases, the number of measurements to estimate $p_{2}$ (a) and $p_{3}$ (b) with accuracy 0.1 is of the order of $100 \times 2^{|A B|}$. 
PT-moments in a trapped-ion quantum simulator.Below, we discuss the experimental demonstration of the measurement of PT moments in a trapped ion quantum simulator. To this end, we evaluate data taken in the context of Ref. [10]. Here, the Rényi entropy growth in quench dynamics was investigated. The system, consisting in total of $N=10$ qubits, was initialized in the Néel state $|\uparrow \downarrow \uparrow \downarrow \ldots\rangle$, and time evolved with

$$
H_{X Y}=\hbar \sum_{i<j} J_{i j}\left(\sigma_{i}^{+} \sigma_{j}^{-}+\sigma_{i}^{-} \sigma_{j}^{+}\right)+\hbar B \sum_{i} \sigma_{i}^{z},
$$

with $\sigma_{i}^{z}$ the third spin-1/2 Pauli operator, $\sigma_{i}^{+}\left(\sigma_{i}^{-}\right)$the spinraising (lowering) operators acting on spin $i$, and $J_{i j} \approx$ $J_{0} /|i-j|^{\alpha}$ the coupling matrix with an approximate power-law decay $\alpha \approx 1.24$ and $J_{0}=420 \mathrm{~s}^{-1}$. After time evolution, randomized measurements were performed, using $M=500$ random unitaries and $P=150$ projective measurements per random unitary.

From these data, PT moments can be inferred [70], with results presented in Fig. 3. For the purity $p_{2}$ (a),(b) we observe good agreement with theory for up to $N=8$ qubits partitions, in particular the raise of $p_{2}$ for partition sizes approaching the total system size which is expected for such nearly pure states. For 9,10-qubit partitions, the data is not shown since the relative statistical error of the estimated
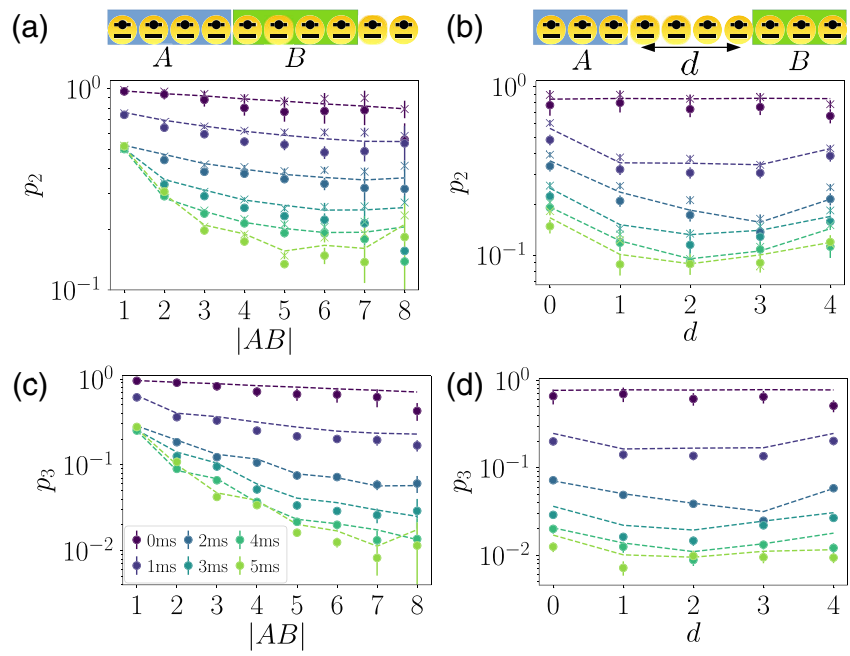

FIG. 3. Reconstruction of $p_{2}=\operatorname{Tr}\left[\left(\rho_{A B}^{T_{A}}\right)^{2}\right]$ and $p_{3}=$ $\operatorname{Tr}\left[\left(\rho_{A B}^{T_{A}}\right)^{3}\right]$ from experimental data [10]. $A$ and $B$ are parts of a total system of 10 qubits. In (a) and (c), we take $A=$ $[1, \ldots,\lfloor|A B| / 2\rfloor]$ and $B=[\lfloor|A B| / 2\rfloor+1, \ldots,|A B|]$. In (b) and (d), we take $A=\{1,2,3\}$ and $B=\{4+d, 5+d, 6+d\}$ with $d=0,1, \ldots 4$. Dots are obtained with the shadow estimator [Eq. (5) second and third order], crosses with the direct estimator (second order) of Ref. [10]. Different colors correspond to different times after the quantum quench with purple [0 $\mathrm{ms}]$ corresponding to the initial product state. For each time, $M=500$ unitaries and $P=150$ measurements per unitary were used. Lines: theory simulation including decoherence [10]. The ratio $p_{2}^{2} / p_{3}$, detecting entanglement according to the $p_{3}$-PPT condition, is shown in Figs. 1(c) and 1(d). data points approaches unity [71]. We, however, note that the measured $\hat{p}_{2}$ is slightly underestimated. This is due to imperfect realizations of the random unitaries, which tend to reduce the estimation of the overlap $\operatorname{Tr}\left(\rho_{r_{1}} \rho_{r_{2}}\right)$. This effect is also present when measuring cross-platform fidelities [34]. For the third PT moment $p_{3}$ (c), (d), we observe the same kind of agreement between theory value and experimental measurements. In particular, at large partition sizes, the protocol is able to measure with high precision small values of $p_{3}$. These small values are indeed fundamental to detect entanglement: a PPT violating state has a negative eigenvalue which reduces the value of $p_{3}$, in comparison with the purity $p_{2}$. This effect is mathematically captured by the $p_{3}$-PPT condition and allowed us to detect PPT violation and thus entanglement for many-body mixed states [see Fig. 1(c)]. In the Supplemental Material [23], we present additional simulations showing the power of the $p_{3}$-PPT condition, in comparison with the negativity and the condition based on purities of nested subsystems.

The third PT moment $p_{3}$ does not only allow to detect mixed-state entanglement. It can also be used to study the dynamics of entanglement in various many-body quantum systems $[22,59-61,65]$. Here, we analyze the behavior of the dimensionless ratio $R_{3}=-\log _{2}\left[p_{3} / \operatorname{Tr}\left(\rho_{A B}^{3}\right)\right]$, which, as shown in quantum field theory, follows the same universal behavior as the negativity during evolution with a local Hamiltonian [61]. We remark that $R_{3}$ is however only well defined for states with $p_{3}>0$ (Werner states in large dimensions are a counterexample [23]). Furthermore, $R_{3}$ is not an entanglement monotone [65]. It vanishes for all product states, but can still be strictly positive for certain separable states $[2,65]$.

Figure 4 illustrates the time evolution of $R_{3}$ for (a) connected and (b) disconnected subsystems $A B$, respectively. The appearing peaks of $R_{3}$ have been predicted and analyzed for various one-dimensional quantum systems subject to local interactions $[61,62]$ (and have also been studied in the context of Rényi mutual information [72,73]). They can be understood in terms of propagating quasi-particles which
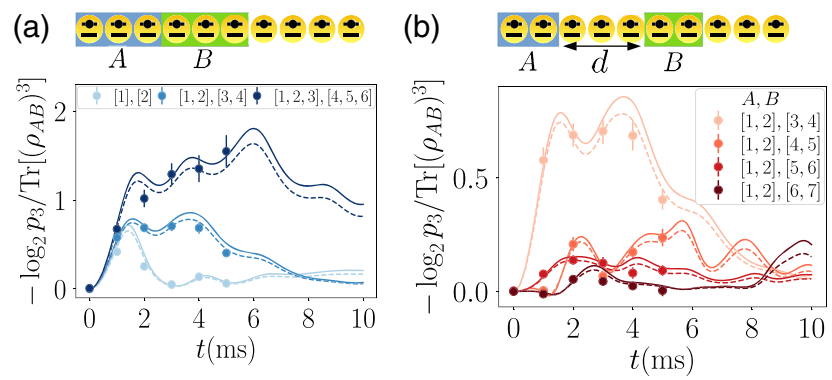

FIG. 4. Evolution of the ratio $R_{3}$ from experimental data [10]. (a) Connected partitions. (b) Disconnected partitions separated by $d=0,1,2,3$ spins. Different colors correspond to different partitions $A B$. Dots are obtained with the shadow estimator Eq. (5) using experimental data [10]. Solid (dashed) lines: theory simulation of unitary dynamics (including decoherence [10]). 
describe collective excitations in the system $[61,62]$. In this picture, entanglement between two partitions $A$ and $B$ is induced by the presence of entangled pairs of quasi-particles shared between $A$ and $B$. For each pair, the individual quasiparticles propagate in opposite directions and start to entangle, in the course of the time evolution, partitions that are more and more separated $[61,62]$. In particular, for two adjacent partitions (a), $R_{3}$ increases at early times, which is consistent with the picture of shared pairs of entangled quasiparticles entering the two partitions immediately. After a certain time $R_{3}$ reaches a maximum and starts to decrease, which can be understood as the time when the quasiparticles start to escape the region $A B$. For separated partitions (b), the peaks are delayed due to the finite speed of propagation of the quasiparticles. In addition, their maximum value is lowered because of the finite lifetimes of quasiparticles. The latter feature is characteristic to chaotic (nonintegrable) thermalizing systems [72] and is in our case further enhanced by decoherence.

Conclusion.-Our protocol extends the paradigm of randomized measurements, yielding the first direct measurement of PT moments in a many-body system. $U$ statistics provides the key ingredient there and enables us to harness a remarkable advantage over state tomography in terms of statistical errors. At a fundamental level, it is therefore natural to investigate how to access new important physical quantities based on random measurement data, and with significant savings in terms of measurement and classical postprocessing over existing methods. This approach can be used to derive protocols to directly infer entanglement measures (including nonpolynomial functions of the density matrix), such as the von-Neumann entropy and the negativity.

We are grateful to Alireza Seif who pointed out interesting error scaling effects for classical shadows in a Scirate comment addressing Ref. [18]. We thank M. Knap, S. Nezami, F. Pollmann, and E. Wybo for discussions and valuable suggestions, as well as M. Joshi for the careful reading and comments on the manuscript. T. Brydges, P. Jurcevic, C. Maier, B. Lanyon, R. Blatt, and C. Roos have generously shared the experimental data of Ref. [10]. Simulations were performed with the QuTiP library [74]. Research in Innsbruck is supported by the European Union's Horizon 2020 research and innovation programme under Grant Agreement No. 817482 (PASQuanS) and No. 731473 (QuantERA via QTFLAG), and by the Simons Collaboration on Ultra-Quantum Matter, which is a grant from the Simons Foundation (651440, P.Z.). B. K. acknowledges financial support from the Austrian Academy of Sciences via the Innovation Fund 'Research, Science and Society', the SFB BeyondC (Grant No. F7107N38), and the Austrian Science Fund (FWF) Grant DKALM: W1259-N27. Research at Caltech is supported by the Kortschak Scholars Program, the U.S. Department of Energy (DE-SC0020290), the U.S. Army Research
Office (W911NF-18-1-0103), and the U.S. National Science Foundation (PHY-1733907). The Institute for Quantum Information and Matter is an NSF Physics Frontiers Center. Research in Trieste is partly supported by European Research Council (Grants No. 758329 and No. 771536) and by the Italian Ministry of Education under the FARE programme. B. V. acknowledges funding from the Austrian Science Foundation (FWF, P. 32597N).

*These authors contributed equally to this work.

[1] J. Preskill, Quantum 2, 79 (2018).

[2] R. Horodecki, P. Horodecki, M. Horodecki, and K. Horodecki, Rev. Mod. Phys. 81, 865 (2009).

[3] J. Eisert, M. Cramer, and M. B. Plenio, Rev. Mod. Phys. 82, 277 (2010).

[4] P. Calabrese and J. Cardy, J. Stat. Mech. (2016) 064003.

[5] R. Horodecki and M. Horodecki, Phys. Rev. A 54, 1838 (1996).

[6] P. Horodecki, Phys. Rev. Lett. 90, 167901 (2003).

[7] R. Islam, R. Ma, P. M. Preiss, M. Eric Tai, A. Lukin, M. Rispoli, and M. Greiner, Nature (London) 528, 77 (2015).

[8] A. M. Kaufman, M. E. Tai, A. Lukin, M. Rispoli, R. Schittko, P. M. Preiss, and M. Greiner, Science 353, 794 (2016).

[9] N. M. Linke, S. Johri, C. Figgatt, K. A. Landsman, A. Y. Matsuura, and C. Monroe, Phys. Rev. A 98, 052334 (2018).

[10] T. Brydges, A. Elben, P. Jurcevic, B. Vermersch, C. Maier, B. P. Lanyon, P. Zoller, R. Blatt, and C. F. Roos, Science 364, 260 (2019).

[11] C. M. Alves and D. Jaksch, Phys. Rev. Lett. 93, 110501 (2004).

[12] A. J. Daley, H. Pichler, J. Schachenmayer, and P. Zoller, Phys. Rev. Lett. 109, 020505 (2012).

[13] J. Cardy, Phys. Rev. Lett. 106, 150404 (2011).

[14] D. A. Abanin and E. Demler, Phys. Rev. Lett. 109, 020504 (2012).

[15] S. J. van Enk and C. W. J. Beenakker, Phys. Rev. Lett. 108, 110503 (2012).

[16] A. Elben, B. Vermersch, M. Dalmonte, J. I. Cirac, and P. Zoller, Phys. Rev. Lett. 120, 050406 (2018).

[17] A. Elben, B. Vermersch, C. F. Roos, and P. Zoller, Phys. Rev. A 99, 052323 (2019).

[18] H.-Y. Huang, R. Kueng, and J. Preskill, Nat. Phys. 16, 1050 (2020).

[19] A. Peres, Phys. Rev. Lett. 77, 1413 (1996).

[20] The partial transpose (PT) operation-acting on subsystem $A$-is defined as $\left(\left|k_{A}, k_{B}\right\rangle\left\langle l_{A}, l_{B}\right|\right)^{T_{A}}=\left|l_{A}, k_{B}\right\rangle\left\langle k_{A}, l_{B}\right|$, where $\left\{\left|k_{A}, k_{B}\right\rangle\right\}$ is a product basis of the joint system $A B$.

[21] G. Vidal and R. F. Werner, Phys. Rev. A 65, 032314 (2002).

[22] P. Calabrese, J. Cardy, and E. Tonni, Phys. Rev. Lett. 109, 130502 (2012).

[23] See Supplemental Material at http://link.aps.org/ supplemental/10.1103/PhysRevLett.125.200501 for a proof and extended discussion of $p_{3}$-PPT condition. This condition is furthermore analyzed in the context of specific states such as Werner states and states generated in quench 
dynamics. Statistical errors of the estimation of PT-moments are discussed in detail, both analytically and numerically, which contains Refs. [24-28].

[24] K. Zyczkowski, Open Syst. Inf. Dyn. 10, 297 (2003).

[25] S. Rana, Phys. Rev. A 87, 054301 (2013).

[26] J. M. Landsberg, Tensors: Geometry and Applications (American Mathematical Society (AMS), Providence, 2012), p. 439.

[27] J. C. Bridgeman and C. T. Chubb, J. Phys. A 50, 223001 (2017).

[28] R. Kueng, Quantum and classical information processing with tensors (lecture notes), 2019), Caltech course notes, https://iqim.caltech.edu/classes.

[29] J. Gray, L. Banchi, A. Bayat, and S. Bose, Phys. Rev. Lett. 121, 150503 (2018).

[30] B. M. Terhal, Phys. Lett. A 271, 319 (2000).

[31] O. Gühne and N. Lütkenhaus, Phys. Rev. Lett. 96, 170502 (2006).

[32] J. Watrous, The Theory of Quantum Information (Cambridge University Press, Cambridge, England, 2018).

[33] X. Mi, B. Vermersch, A. Elben, P. Roushan, Y. Chen, P. Zoller, and V. Smelyanskiy, APS March Meeting 2020, Vol. 65, http:// meetings.aps.org/Meeting/MAR20/Session/U16.6.

[34] A. Elben, B. Vermersch, R. van Bijnen, C. Kokail, T. Brydges, C. Maier, M. K. Joshi, R. Blatt, C. F. Roos, and P. Zoller, Phys. Rev. Lett. 124, 010504 (2020).

[35] M. K. Joshi, A. Elben, B. Vermersch, T. Brydges, C. Maier, P. Zoller, R. Blatt, and C. F. Roos, Phys. Rev. Lett. 124, 240505 (2020).

[36] E. Cornfeld, E. Sela, and M. Goldstein, Phys. Rev. A 99, 062309 (2019).

[37] Y. Zhou, P. Zeng, and Z. Liu, Following Letter, Phys. Rev. Lett. 125, 200502 (2020).

[38] C. Dankert, R. Cleve, J. Emerson, and E. Livine, Phys. Rev. A 80, 012304 (2009).

[39] Y. Nakata, C. Hirche, M. Koashi, and A. Winter, Phys. Rev. X 7, 021006 (2017).

[40] B. Vermersch, A. Elben, M. Dalmonte, J. I. Cirac, and P. Zoller, Phys. Rev. A 97, 023604 (2018).

[41] D. Gross, Y.-k. Liu, S. T. Flammia, S. Becker, and J. Eisert, Phys. Rev. Lett. 105, 150401 (2010).

[42] M. Cramer, M. B. Plenio, S. T. Flammia, R. Somma, D. Gross, S. D. Bartlett, O. Landon-Cardinal, D. Poulin, and Y.-K. Liu, Nat. Commun. 1, 149 (2010).

[43] G. Torlai, G. Mazzola, J. Carrasquilla, M. Troyer, R. Melko, and G. Carleo, Nat. Phys. 14, 447 (2018).

[44] M. Gu, J. Kahn, R. Kueng, and J. A. Tropp, J. Phys. A 53, 204001 (2020).

[45] J. Haah, A. W. Harrow, Z. Ji, X. Wu, and N. Yu, IEEE Trans. Inf. Theory 63, 5628 (2017).

[46] R. O'Donnell and J. Wright, in Proceedings of the FortyEighth Annual ACM Symposium on Theory of Computing, STOC '16 (ACM, New York, 2016), pp. 899-912.

[47] B. Vermersch, A. Elben, L. M. Sieberer, N. Y. Yao, and P. Zoller, Phys. Rev. X 9, 021061 (2019).

[48] A. Elben, J. Yu, G. Zhu, M. Hafezi, F. Pollmann, P. Zoller, and B. Vermersch, Sci. Adv. 6, eaaz3666 (2020).

[49] Z.-P. Cian, H. Dehghani, A. Elben, B. Vermersch, G. Zhu, M. Barkeshli, P. Zoller, and M. Hafezi, arXiv:2005.13543.

[50] M. C. Tran, B. Dakić, F. m. c. Arnault, W. Laskowski, and T. Paterek, Phys. Rev. A 92, 050301(R) (2015).
[51] M. C. Tran, B. Dakić, W. Laskowski, and T. Paterek, Phys. Rev. A 94, 042302 (2016).

[52] A. Ketterer, N. Wyderka, and O. Gühne, Phys. Rev. Lett. 122, 120505 (2019).

[53] W.-H. Zhang, C. Zhang, Z. Chen, X.-X. Peng, X.-Y. Xu, P. Yin, S. Yu, X.-J. Ye, Y.-J. Han, J.-S. Xu, G. Chen, C.-F. Li, and G.-C. Guo, Phys. Rev. Lett. 125, 030506 (2020).

[54] L. Knips, J. Dziewior, W. Kobus, W. Laskowski, T. Paterek, P. J. Shadbolt, H. Weinfurter, and J. D. A. Meinecke, npj Quantum Inf. 6 (2020).

[55] P. Calabrese, J. Cardy, and E. Tonni, J. Stat. Mech. (2013) P02008.

[56] P. Ruggiero, V. Alba, and P. Calabrese, Phys. Rev. B 94, 035152 (2016).

[57] Y. Javanmard, D. Trapin, S. Bera, J. H. Bardarson, and M. Heyl, New J. Phys. 20, 083032 (2018).

[58] X. Turkeshi, P. Ruggiero, and P. Calabrese, Phys. Rev. B 101, 064207 (2020).

[59] C.-M. Chung, V. Alba, L. Bonnes, P. Chen, and A. M. Läuchli, Phys. Rev. B 90, 064401 (2014).

[60] K.-H. Wu, T.-C. Lu, C.-M. Chung, Y.-J. Kao, and T. Grover, Phys. Rev. Lett. 125, 140603 (2020).

[61] A. Coser, E. Tonni, and P. Calabrese, J. Stat. Mech. (2014) P12017.

[62] V. Alba and P. Calabrese, Europhys. Lett. 126, 60001 (2019).

[63] J. Kudler-Flam, M. Nozaki, S. Ryu, and M. T. Tan, J. High Energy Phys. 01 (2020) 031.

[64] V. Alba and F. Carollo, arXiv:2002.09527.

[65] E. Wybo, M. Knap, and F. Pollmann, Phys. Rev. B 102, 064304 (2020).

[66] D. Gross, K. Audenaert, and J. Eisert, J. Math. Phys. (N.Y.) 48, 052104 (2007).

[67] M. Ohliger, V. Nesme, and J. Eisert, New J. Phys. 15, 015024 (2013).

[68] M. Paini and A. Kalev, arXiv:1910.10543.

[69] W. Hoeffding, in Breakthroughs in Statistics (Springer, New York, 1992), pp. 308-334.

[70] Theoretically, an ideal distribution of the measurement budget $M \cdot P$ would consist in setting $P=1$, i.e., sampling new random unitaries for each experimental run. Experimentally, it might be, however, beneficial to use $P \geq 1$. In this situation, we replace the estimators defined in Eq. (3) with $\hat{\rho^{(r)}}=\sum_{s=1}^{P} \hat{\rho}^{(r, s)}$, where $\hat{\rho}^{(r, s)}=\underset{i}{\bigotimes}\left(3\left(u_{i}^{(r)}\right)^{\dagger}\left|k_{i}^{(r, s)}\right\rangle\right.$ $\left.\left\langle k_{i}^{(r, s)}\right| u_{i}^{(r)}-\mathbb{I}_{2}\right)$ and $k_{i}^{(r, s)}$ is the outcome of the measurement $s$ obtained after the application of the unitary $r$.

[71] The distribution of the measurement budget $M \cdot P$ in Ref. [10] into unitaries $M$ and projective measurements per unitary $P$ has been optimized for the purity estimator $\tilde{p}_{2}$ presented in Ref. [10], which differs from $\hat{p}_{2}$ defined in Eq. (5). Thus, for the present dataset [10] with $M=500$ and $P=150$, the statistical uncertainty of the $\tilde{p}_{2}$ is smaller than for $\hat{p}_{2}$, which performs best for $P=\mathcal{O}(1)$ and a correspondingly larger number of unitaries $M$.

[72] V. Alba and P. Calabrese, Phys. Rev. B 100, 115150 (2019).

[73] S. Maity, S. Bandyopadhyay, S. Bhattacharjee, and A. Dutta, Phys. Rev. B 101, 180301(R) (2020).

[74] J. Johansson, P. Nation, and F. Nori, Comput. Phys. Commun. 184, 1234 (2013). 